\title{
Intraoperative Awareness during General Anesthesia: Experience in 200 Patients in “185's Hospital for Emergency Surgeries and Burn". (An Observational Questionnaire-based Study)
}

\author{
Eslam Ragab Heggy*, Sherif Mamdouh Abbas, Atef Glal Abd El Mawla, Mina Adolf, Ahmed Fetouh \\ Cairo University, Cairo, Egypt
}

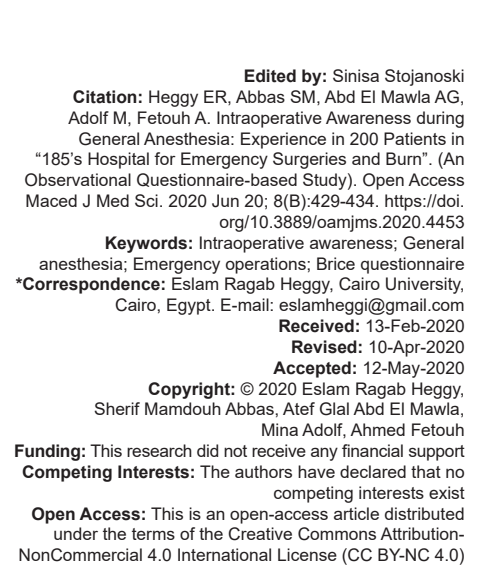

\begin{abstract}
BACKGROUND: General anesthesia (GA) is a complex state of hypnosis, amnesia, and suppression of stress response to stimuli and production of a quiet surgical field. Awareness under GA considered as unexpected and undesirable complication which can be source of pain and torture for many individuals after surgery.

AIM: The aim of the study was to evaluate the incidence of intraoperative awareness during general anesthesia in emergency operations using Brice questionnaire.

METHODS: Two hundred patients were included and undergone emergency operations under GA at Cairo University's Hospitals. Intravenous induction then inhalational maintenance of anesthesia with muscle relaxant was commenced, basic vital signs were monitored and kept within normal range. Depth of anesthesia was manipulated and kept adequate intraoperatively according to patient's clinical status. At the end of surgery, patients were fully reversed and extubated fully awake then transferred to the recovery room, then data were collected.

RESULTS: Data analysis showed $110(55 \%)$ men and 90 (45\%) women. Two hours postoperatively, one patient $(0.5 \%)$ reported intraoperative awareness, while 199 patients $(99.5 \%)$ remembered nothing intraoperatively.

CONCLUSION: The incidence of intraoperative awareness in our emergency hospital is relatively fair and clinica evaluation of anesthesia depth is an effective measure to detect and prevents intraoperative awareness.
\end{abstract}

\section{Introduction}

The purpose of anesthesia is to induce unconsciousness through administration of drugs. Therefore, it is fundamental to provide patients with analgesia, anxiolysis, amnesia and suppression of hormonal, cardiocirculatory, and motor responses in the surgical stress setting [1].

Prys-Roberts [2] defined anesthesia as the state in which (as a result of drug induced unconsciousness) the patient neither perceives nor recalls noxious stimuli. He further stated that analgesia, muscle relaxation, and suppression of autonomic activity are not the components of anesthesia, but should be considered as desirable supplements to the state of anesthesia as a means to enable surgery to be performed [2].

Intraoperative awareness during general anesthesia (GA) is the second most common concern of patients after post-operative vomiting [3].

The causes for this event are frequently a consequence of inadequate anesthesia technique, device failure, addicted patients, excessive use of neuromuscular blocking agents, and inadequate monitoring [4].
Awareness during anesthesia with intraoperative memory occurs when the patient is able to process information and produces specific responses to several stimuli [5].

The different phases of intraoperative awareness or memory are independent. Explicit or declarative memory is when the patient remembers facts, events, or experiences that occurred during GA [6].

Regarding implicit or procedural memory defined as the memory of motor and sensorial capacities and abilities, the patient is unable to verbally express his experience during anesthesia, but there are changes in his post-operative behavior, habits, and performance in such a way that psychological tests are required to detect the implicit memory [5].

Another intraoperative awareness phase is the state of wakefulness, when the patient is able to react to stimuli during surgery, but does not recall nor is conscious of the experienced reactions [7].

The experience of consciousness is not the same for all patients. They may be collected as memories (hearing, tactile sensation, paralysis sensation, and difficulty to move and breath, helplessness sensation, panic, anxiety, chronic fear and medical fear, insomnia, and recurrent nightmares), and neurosis, known as 
post-traumatic stress disorder and requiring psychiatric treatment [8].

An awareness incidence of $1.2 \%$ has been documented in 1960, but recent studies show an incidence of $0.1-0.2 \%$, which may change according to biological types, anesthetic drugs, and applied surgical procedures [9].

A multicenter study with 19,575 patients showed an intraoperative awareness incidence of $0.13 \%$ [10].

One approach to understand the critical mechanisms by which general anesthetics suppress awareness is to seek for invariant changes in the human brain as patients lose and regain consciousness under the effects of a variety of anesthetic agents. Such information is increasingly available from a variety of electrophysiological instruments that have been developed such as bispectral index (BIS) monitor, Evoked potentials, Patient State Analyzer monitor, and The dimensionless Narcotrend index monitor [11].

\section{Patients and Methods}

The study design was approved by ethics and research committee of Anesthesiology Department, Faculty of Medicine, Cairo University. The study included 200 patients. All of them were scheduled for surgical procedures under general anesthesia at "185 hospital for emergency operations and burn" Kasr Al Ainy Teaching Hospital, Cairo University.

\section{Inclusion criteria}

All patients older than 16 years old, undergoing emergency operations and balanced, controlled GA were included in the study.

\section{Exclusion criteria}

Patients undergoing their operations under total intravenous anesthesia, or patients with neuropsychological disorders preventing them from responding to the questionnaire were excluded from the study.

The anesthesia of all cases was performed by residents who were supervised by specialists. The choice of the drugs of premedication and inhalational anesthetic for a given surgical procedure depended on the anesthetist's clinical experience, the patient's condition, and the type of surgery.

All individuals had intravenous induction either by propofol ( $2 \mathrm{mg} / \mathrm{kg}$ ) or thiopental sodium $(5 \mathrm{mg} / \mathrm{kg}$ ) and volatile maintenance of anesthesia using either sevoflurane with minimum alveolar concentration (MAC) ranging from $1 \%$ to $1.7 \%$ or isoflurane with MAC ranging from $0.8 \%$ to $1.2 \%$. Atracurium $(0.1 \mathrm{mg} / \mathrm{kg})$ was used as a muscle relaxant of maintenance in all patients.

In all patients, vital signs (heart rate, blood pressure, and $\mathrm{O}_{2}$ saturation) were monitored during anesthesia and were kept within normal range.

The depth of anesthesia was controlled intraoperatively based on the patient's clinical status such as heart rate, blood pressure, alimentary signs (e.g., swallowing), and skin signs (e.g., sweating). All the studied patients were kept at adequate level of anesthesia. Instrumental monitoring of anesthesia depth was not carried out.

At the end of the surgical procedure, all patients were fully reversed and extubated fully awake then transferred to the recovery room.

The following data were collected:

- $\quad$ Gge
- $\quad$ Type of operation
- $\quad$ ASA classification
The drugs of premedication, induction and
maintenance of anesthesia.

The incidence of intraoperative awareness was assessed using Brice questionnaire [12] which was modified for the purposes of our study; i.e., the questions for patients were extended to encourage them to provide answers that were more descriptive. The questionnaire was structured similar to questionnaires used in other studies focusing on the detection of intraoperative awareness [13], [14].

Data were collected for all participants by residents who were involved in the survey through a data sheet. They visited the patients $2 \mathrm{~h}$ postoperatively and after conversations with the patients, they completed the questionnaire, which was subsequently verified by anesthesiologists in charge of assessing intraoperative awareness. The following questions were included:

1. What was the last thing you remembered happening before you went to sleep?

2. What was the first thing you remembered happening on waking?

3. Did you dream or have any other experiences while you were asleep?

4. What was the worst thing about your operation?

5. What was the next worst thing?

The patients' reports were classified according to the classifications commonly used in the literature [14], [15] - as follows:

Definite awareness $(A)$ : A recalled event occurring during anesthesia or surgery that was confirmed by the attending personnel present in the operating room was considered awareness. 
Possible awareness (B): A situation in which the patient was unable to remember any particular event during anesthesia or surgery, yet memories could have been associated with the surgical procedure was defined as possible awareness.

No awareness (C): A lack of recalled intraoperative events with probable memories of situations associated with the immediate pre-induction or post-operative period.

\section{Results}

Data from 200 patients were analyzed, including $110(55 \%)$ men and 90 (45\%) women. The median age of the participants was 44 years. The physical status of the participants was assessed as follows: ASA I: 132 (66\%); ASA II: 60 (30\%); and ASA III: 8 (4\%).

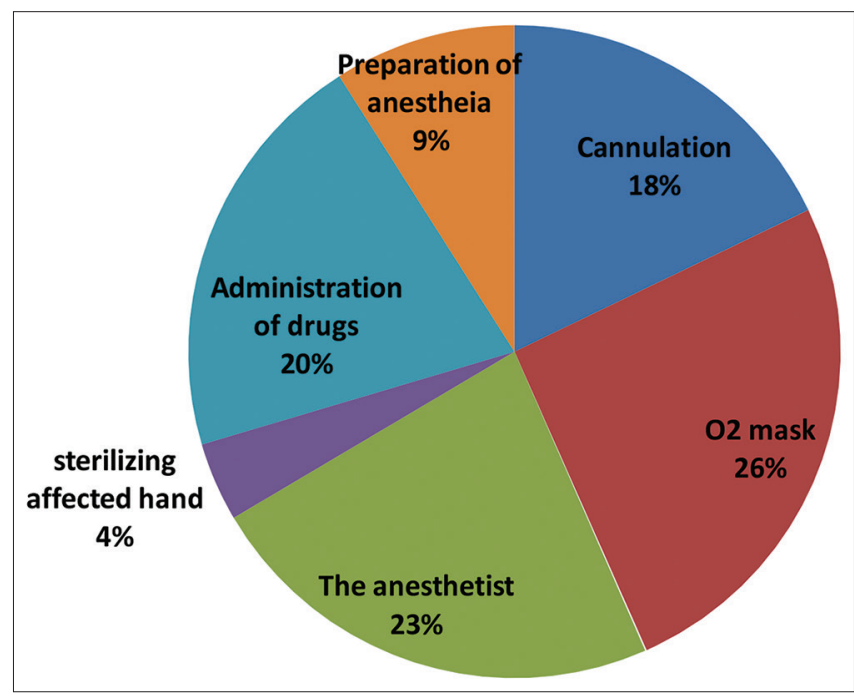

Figure 1: Events remembered by patients before going to sleep

None of the patients was premeditated with benzodiazepines. Other drugs (e.g., famotidine and metoclopramide) were administered to 145 $(72.5 \%)$ patients, and five $(2.5 \%)$ individuals were not pharmacologically pre-medicated. There were no data concerning premedication in $50(25 \%)$ cases.

Two hours postoperatively, there was one event $(0.5 \%)$ that could be classified as intraoperative awareness (category A). This patient was a 26 years old man without previous surgical history whose preoperative physical status was assessed as ASA I. The patient underwent lumbar spinal fixation at level of L1-L2. Anesthesia was induced intravenously by propofol (2 $\mathrm{mg} / \mathrm{kg})$ and maintained with inhalational isoflurane (MAC 1.2\%).

When was he asked about the last thing, he remembered happening before going to sleep, $\mathrm{He}$ cried and said "I felt my head was tilted, something obstructing my throat, I cannot take my breath nor move my head and I heard somebody said" " pull him down." On asking the resident who anaesthetized him, he confirmed the words of the patient. His report was classified as definitive awareness during anesthesia (category A).

The remaining patients (99.5\%) did not remember anything between going to sleep and waking up.

According to the events remembered by patients before going to sleep, 48 patients (24\%) remembered nothing. The data of the remaining 151 patients $(76 \%)$ are explained in Figure 1.

According to the events remembered by the patients after recovery from anesthesia, 36 patients $(18 \%)$ remembered nothing. The data from the remaining 163 patients (82\%) are explained in Figure 2.

According to unpleasant events connected with surgery and anesthesia, 63 patients (32\%) experienced no unpleasant events. The data from the remaining 136 patients $(68 \%)$ are explained in Figure 3.

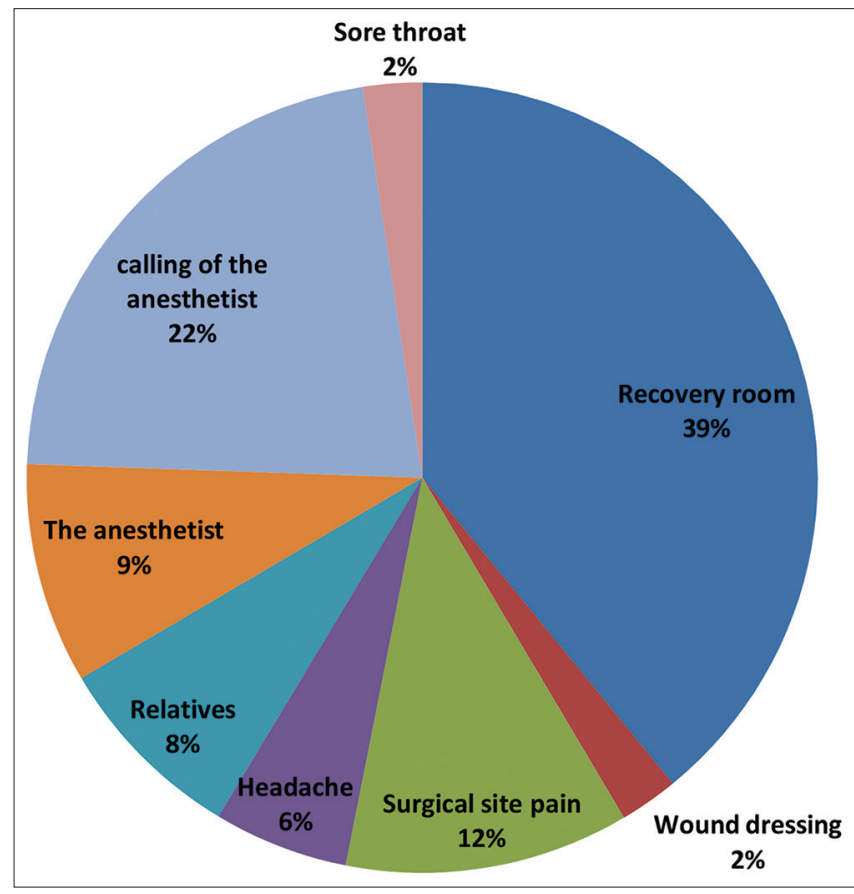

Figure 2: Events remembered by patients after recovery from anesthesia

\section{Discussion}

Intraoperative awareness is a subjective sensation, whereas verification and classification of patient's reports are based on the criteria selected by the physician assessing the event.

In this analysis, we used the recommendations that are most commonly accepted in the literature [5], [10], [13]. According to these criteria, patients with perioperative recall can be divided into those who recall concrete events associated with the surgery (awareness) and those who 
cannot describe their sensations precisely enough to consider them as fragments of events that occurred during surgery [16], [17].

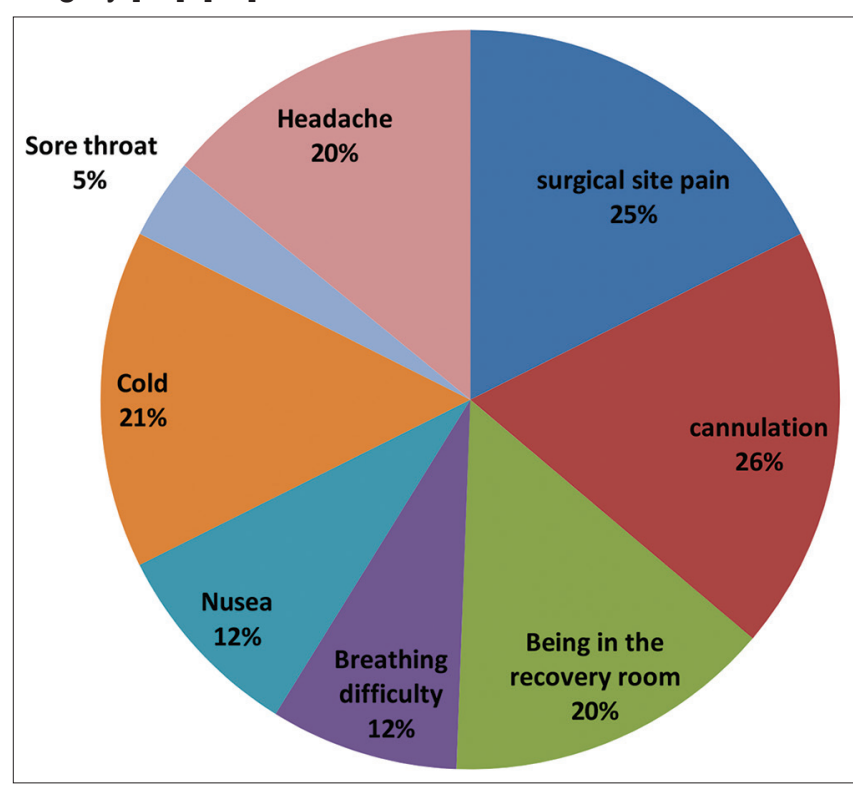

Figure 3: Unpleasant events connected with surgery and anesthesia remembered by patients

In this study, the incidence of accidental awareness during GA(AAGA) was one case of definitive intraoperative awareness (1:200). Considering the size of the population studied and the incidence of intraoperative awareness reported by other authors, the result is likely fair.

The studies using the same methodology of this study which is the basis of the Brice and colleagues, consistently report the incidence of AAGA as 1-2:1000 but estimates still vary considerably from 1:100 to 1:4000 [18].

The studies that used Brice-style patient questioning twice over a $48 \mathrm{~h}$ period yielded a much lower incidence of 1:14 500 [19].

In contrast, studies using instruments without specific questions pertaining to awareness such as Pollard et al. [20], quality assurance data such as Mashour et al. [21] or spontaneous reports such as the recent National Audit Project (NAP) five have consistently found the incidence to be lower [21].

It was unclear from these conflicting reports whether the differences in incidence resulted from disparities in patient population, anesthetic technique, clinical severity, or method of detection [5].

In an attempt to resolve the controversy across studies and study populations, Mashour et al. [5] compared the incidence of intraoperative awareness with explicit recall in a single population of surgical patients who received both a standard post-operative evaluation (without a structured interview intended to detect awareness) and a single modified Brice interview at 30 days [5].

The modified Brice interview detected 19 instances of definite intraoperative awareness with explicit recall in approximately 19,000 surgical patients [5].
Although the modified Brice interview cannot be regarded as a "gold-standard" psychometric test for awareness and memory, it has been associated consistently with a higher incidence compared with alternative methods.

Intraoperative awareness is more common in patients undergoing total intravenous anesthesia [13] and those who receiving muscle relaxants [22].

The determinations ofanestheticconcentrations during intravenous anesthesia in the real-time are not possible. In this study, patients undergoing total intravenous anesthesia were not included.

Clear and consistent findings have emerged from the five major randomized controlled trials focused on the BIS [23]. Some of these trials demonstrated that the BIS monitor was effective in reducing definite awareness events compared with routine clinical care in patients at high risk for the complication; this has also been demonstrated for patients receiving total I.V. anesthesia [6], [24].

In contrast, another trial demonstrated that alarms based on the BIS are not superior to alarms based on end-tidal anesthetic concentration in preventing awareness with explicit recall in patients at high risk for the complication [25].

In 2012, a large randomized clinical trial was published involving 30,000 people. It has been shown that in patients anesthetized without the use of instrumental monitoring, and without the use of standardized (based on MAC) protocol anesthesia, definite, and possible intraoperative awareness during anesthesia occurs almost five-fold higher [5].

Despite the advantages of modern general anesthetic agents over the past four decades, with less cardiovascular depression and rapid elimination, the practice of pharmacological paralysis with limited hypnotic administration continued to be popular and still has proponents in modern practice [26].

In the observational study by Sandin et al. [27], the incidence of unintended awareness among patients who received GA without neuromuscular blocking agents was $0.1 \%$, compared with $0.18 \%$ when patients were pharmacologically paralyzed [27].

This important insight has again been corroborated in the recently published NAP five study [28], where the overwhelming majority of awareness reports were from patients who had received neuromuscular blocking drugs and also where the anesthetic concentration was reduced toward the end of surgery before antagonizing neuromuscular blockade. The avoidance or minimization of pharmacological paralysis might be the most effective currently available method to prevent traumatic intraoperative awareness [28].

Detection of intraoperative awareness is not easy because it depends on patient reports rather than objective measures. Prospective methods using structured questionnaires detect substantially 
more awareness events than approaches based on spontaneous patient reports [5].

However, a concern regarding the questions in the Brice questionnaire is that they have not been psychometrically validated and might have the potential to elicit false reports or memories [21]. This latter possibility is consistent with the finding that a significant proportion of patients only report awareness at later time points after multiple structured interviews [21].

Regardless of the detection method, distinguishing true from false awareness reports is difficult. Occasionally, a patient report is so detailed and specific in describing intraoperative experiences, events, or discussions that independent arbiters can concur that awareness definitely occurred [5]. Commonly, however, patient reports are vague and experts express divergent opinions regarding whether or not a patient was truly aware [23]. If many of the possible awareness reports do represent true awareness, this would mean that the incidence of intraoperative awareness has been even higher than studies have suggested.

In contrast to possible awareness experiences, it is important to clarify that most reports of intraoperative dreaming, which was previously viewed as possible or near awareness experiences, is likely to be unrelated to intraoperative awareness and does not necessarily indicate that patients were insufficiently anaesthetized during surgery [29].

Basedontheclinicalandelectroencephalographic evidence, it is possible that dreaming occurs during emergence from GA, when patients are sedated or in a physiological sleep state [29]. However, Samuelsson et al. [30] found that, while the content of dreams was unrelated to awareness, the incidence of intraoperative awareness was 19 times more common among patients who reported a dream after surgery. Therefore, the precise relationship between awareness and dreaming remains unresolved [30].

\section{Some limitations}

Conversations with patients in the post-operative period were carried out only once and at various time intervals following the completion of anesthesia. In the majority of studies, such assessments are performed twice or 3 times: In the operating room, on day 1-3 after anesthesia and 2-4 weeks after anesthesia which is likely to increase the detection rates of adverse events [6], [14].

We are not arguing that further research is unnecessary in these aspects of the field, but rather that new studies with disparate results do not necessarily create "controversy" unless the methodology is clearly superior and results are particularly novel compared with the existing literature.

\section{Conclusion}

The results of the present study show that the incidence of intraoperative awareness in 185 hospital for emergency surgeries and burn is relatively fair and clinical evaluation of anesthesia depth is an effective measure to detect and prevents intraoperative awareness.

It was found that there was considerable number of patients with post-operative.

Un-pleasant sensations such as surgical site pain, pre-operative cannulation pain and being in the recovery room for a long time. These unpleasant events need more attention from the anesthesiologists to improve the anesthesia care.

\section{References}

1. Pandit JJ, Andrade J, Bogod DG, Hitchman JM, Jonker WR, Lucas $\mathrm{N}$, et al. $5^{\text {th }}$ national audit project (NAP5) on accidental awareness during general anaesthesia: Protocol, methods, and analysis of data. $\mathrm{Br} \mathrm{J}$ Anaesth 2014;113(4):540-8. https://doi. org/10.1093/bja/aeu312

PMid:25204695

2. Tempe DK. In seach of a reliable a warness monitors. Anesth Analg 2001;92(4):801-4.

PMid:11273904

3. Macario A, Weinger M, Carney S, Kim A. Which clinica anesthesia outcomes are important to avoid? The perspective of patients. Anesth Analg 1999;89(3):652-8. https://doi. org/10.1093/bja/aeu312

PMid:10475299

4. Ghoneim MM. Awareness during anesthesia. Anesthesiology 2000;92(2):597-602.

PMid: 10691248

5. Mashour GA, Shanks A, Tremper KK, Kheterpal S, Turner $\mathrm{CR}$, Ramachandran SK, et al. Prevention of intraoperative awareness with explicit recall in an unselected surgical population: A randomized comparative effectiveness trial. Anesthesiology 2012;117(4):717-25. https://doi.org/10.1213/ ane.0b013e318281e9ad

PMid:22990178

6. Avidan MS, Jacobsohn E, Glick D, Burnside BA, Zhang L, Villafranca $A$, et al. Prevention of intra operative awareness in a high-risk surgical population. N Engl J Med 2011;365(7):591-600. PMid:21848460

7. Sanders RD, Tononi G, Laureys S, Sleigh JW. Unresponsiveness versus unconsciousness. Anesthesiology 2012;116(4):946-59. https://doi.org/10.1097/aln.0b013e318249d0a7

PMid:22314293

8. Myles PS, Williams DL, Hendrata M, Anderson H, Weeks AM. Patient satisfaction after anaesthesia and surgery: Results of a prospective survey of 10,811 patients. $\mathrm{Br} \mathrm{J}$ Anaesth 2000;84(1):6-10.

PMid: 10740539

9. Mashour GA, Esaki RK, Tremper KK, Glick DB, O'Connor M, Avidan MS. A novel classification instrument for intraoperative 
awareness events. Anesth Analg 2010;110(3):813-5. https://doi. org/10.1213/ane.0b013e3181b6267d

PMid:19713251

10. Sebel BS, Bowdle A, Ghoneim MM, Rampil IJ, Padilla RE, Gan TJ, et al. The incidence of awareness during anesthesia: A multicentrer United States Study. Anesth Analg 2004;99(3):8339. https://doi.org/10.1213/01.ane.0000130261.90896.6c PMid:15333419

11. Ghoneim MM, Weiskopf, Richard B. Awaerness during anesthesia clinical concept and commentary. Anesthesiology 2000;92(2):597. PMid:10691248

12. Mashour GA, Tremper KK, Avidan MS. Protocol for the "Michigan Awareness Control Study": A prospective, randomized, controlled trial comparing electronic alerts based on bispectral index monitoring or minimum alveolar concentration for the prevention of intraoperative awareness. BMC Anesthesiol 2009;9:7. https://doi.org/10.1186/1471-2253-9-7

PMid:19891771

13. Morimoto $\mathrm{Y}$, Nogami $\mathrm{Y}$, Harada K, Tsubokawa T, Masui K. Awareness during anesthesia: The results of a questionnaire survey in Japan. J Anesth 2011;25(1):72-7. https://doi. org/10.1007/s00540-010-1050-y PMid:21153846

14. $\mathrm{Xu} \mathrm{L,} \mathrm{Wu} \mathrm{AS,} \mathrm{Yue} \mathrm{Y.} \mathrm{The} \mathrm{incidence} \mathrm{of} \mathrm{intra-operative} \mathrm{awareness}$ during general anesthesia in China: A multi-center observational study. Acta Anaesthesiol Scand 2009;53(7):873-82. https://doi. org/10.1111/j.1399-6576.2009.02016.x PMid:19496761

15. Ghoneim MM, Block RI, Haffarnan M, Mathews MJ. Awareness during anesthesia: risk factors, causes and sequelae: A review of reported cases in the literature. Anesth Analg 2009;108(2):52735. https://doi.org/10.1213/ane.0b013e318193c634 PMid:19151283

16. Avidan MS, Zhang L, Burnside BA, Finkel KJ, Searleman AC, Selvidge JA, et al. Anesthesia awareness and the bispectral index. N Engl Med 2008;358(11):1097-8. https://doi. org/10.1213/01.ane.0000130261.90896.6c PMid: 18337600

17. Mahla ME, Black S, Cucchiara RF. Neurologic monitoring In: Miller RD, editor. Anaesthesia. $6^{\text {th }}$ ed., Vol. 38. New York: Churchill Livingstone; 2005. p. 1511-50.

18. Ye Z, Guo QL, Zheng $H$. Investigation and analysis of the incidence of awareness during general anesthesia. Zhong Nan Da Xue Xue Bao Yi Xue Ban 2008;33(6):533-6. PMid:18600003

19. Pollard RJ, Coyle JP, Gilbert RL, Beck JE. Intraoperative awareness in a regional medical system: A review of 3 years' data. Anesthesiology 2007;106(2):269-74.

PMid: 17264720

20. Mashour GA, Wang LY, Turner CR, Vandervest JC, Shanks A, Tremper KK. A retrospective study of intraoperative awareness with methodological implications. AnesthAnalg 2009;108(2):5216. https://doi.org/10.1097/00000542-200702000-00014 PMid:19151282
21. Mashour GA, Kent C, Picton P, Ramachandran SK Tremper KK, Turner CR, et al. Assessment of intraoperative awareness with explicit recall: A comparison of 2 methods. Anesth Analg 2013;116(4):889-91. https://doi.org/10.341 0/f.718775534.793505509

\section{PMid:23460567}

22. Errando CL, Sigl JC, Robles M, Calabuig E, Garcia J, Arocas F, et al. Awareness with recall during general anaesthesia: A prospective observational evaluation of 4001 patients. $\mathrm{Br} J$ Anaesth 2008;101(2):178-85. https://doi.org/10.1097/ aln.0b013e31826904a6

PMid:18515816

23. Myles PS, Leslie K, McNeil J, Forbes A, Chan MT Bispectral index monitoring to prevent awareness during anaesthesia: The B-aware randomised controlled trial. Lancet 2004;363(9423):1757-63. https://doi.org/10.1093/bja/aen144 PMid:15172773.

24. Zhang C, Xu L, Ma YQ, Sun YS, Li YH, Zhang L, et al. Bispectral index monitoring prevent awareness during total intravenous anesthesia: A prospective, randomized, double-blinded, multicenter controlled trial. Chin Med J (Engl) 2011;124(22):3664-9. https://doi.org/10.1016/s0140-6736(04)16300-9

\section{PMid:22340221}

25. Punjasawadwong $\mathrm{Y}$, Phongchiewboon $\mathrm{A}$, Bunchungmongkol $\mathrm{N}$ Bispectral index for improving anaesthetic delivery and postoperative recovery. Cochrane Database Syst Rev 2014;6(1):CD003843. https://doi.org/10.1056/nejmoa1100403 PMid:24937564

26. Pandit JJ, Andrade J, Bogod DG, Hitchman JM, Jonker WR, Lucas $\mathrm{N}$, et al. $5^{\text {th }}$ National audit project (NAP5) on accidental awareness during general anaesthesia: Summary of main findings and risk factors. $\mathrm{Br} \mathrm{J}$ Anaesth 2014;113(4):549-59. https://doi.org/10.1213/ane.0b013e3181732b0c PMid:25204697

27. Sandin RH, Enlund G, Samuelsson P, Lennmarken C. Awareness during anaesthesia: A prospective case study. Lancet 2000;355(9205):707-11. https://doi.org/10.1002/14651858. cd003843.pub2 PMid: 10703802

28. Cook TM, Andrade J, Bogod DG, Hitchman JM, Jonker WR, Lucas N, et al. $5^{\text {th }}$ National Audit Project (NAP5) on accidental awareness during general anaesthesia: Patient experiences, human factors, sedation, consent, and medicolegal issues. $\mathrm{Br} J$ Anaesth 2014;113(4):560-74. https://doi.org/10.1016/ s0140-6736(99)11010-9 PMid:25204696

29. Leslie K, Skrzypek H, Paech MJ, Kurowski I, Whybrow T. Dreaming during anesthesia and anesthetic depth in elective surgery patients: A prospective cohort study. Anesthesiology 2007;106(1):33-42. https://doi.org/10.1093/bja/aeu314 PMid: 17197843

30. Samuelsson P, Brudin L, Sandin RH. Intraoperative dreams reported after general anaesthesia are not early interpretations of delayed awareness. Acta Anaesthesiol Scand 2008;52(6):8059. https://doi.org/10.1097/00000542-200701000-00010 PMid:18477084 